\title{
Organisationen verändern
}

Neue digitale Technologien führen in vielen Bereichen zu tiefgreifenden Veränderungen in den Arbeits- und Organisationsformen. Im folgenden Kapitel geht es darum, wie sich der daraus resultierende organisationale Wandel erfolgreich gestalten lässt. Im ersten Beitrag von Appelt, Allmendinger und Bronner wird darauf eingegangen, wie sich diese Veränderungen und insbesondere die agilen Arbeitsweisen auf die Rolle des Human Resource Managements auswirken. Da dieser Bereich oft für die Etablierung solcher Veränderungen in der gesamten Organisation verantwortlich ist, lassen sich hier viele für den digitalen Wandel relevanten Themen exemplarisch darstellen. Neben solchen Veränderungen in der Arbeitsweise, spielt beim digitalen Wandel auch die Veränderung der Führungskultur eine wichtige Rolle. Im Beitrag von Kurt wird dies am Beispiel des Holacracy-Konzeptes dargestellt. In diesem Beitrag wird erläutert, wie sich Holacracy als Führungsprinzip in Organisationen etablieren lässt und welche Herausforderungen sich dabei ergeben. Neben der Beschäftigung mit den Herausforderungen, die durch neue Organisations- und Führungsformen entstehen, liegt ein weiterer Schwerpunkt dieses Kapitels in der Frage, wie sich die Arbeit einzelner Unternehmensbereiche durch die Verwendung von neuen analytischen Verfahren und Informationssystemen verändert. Der Beitrag von Thums und Müller geht dieser Frage am Beispiel des Human Resource Managements nach. Stransky, Reder, Huber und Hauer stellen sich im letzten Beitrag dieses Kapitels dann die Frage, wie diese neuen analytischen Verfahren und Möglichkeiten die Rolle des Controllers in der Zukunft verändern werden.

(C) Springer Fachmedien Wiesbaden GmbH, ein Teil von Springer Nature 2019

A. Lochmahr, P. Müller, P. Planing, T. Popović, Digitalen Wandel gestalten

https.//doi.org/10.1007/978-3-658-24651-8_3.0 